\title{
TOXICITY OF DIETARY METHYLMERCURY TO FISH: DERIVATION OF ECOLOGICALLY MEANINGFUL THRESHOLD CONCENTRATIONS
}

\author{
David C. Depew, *† Niladri Basu, $\ddagger$ Neil M. Burgess, $§$ linda M. Campbell,$\dagger$ Ed W. Devlin, $\|$ Paul E. Drevnick,\# \\ Chad R. Hammerschmidt, †† Cheryl A. Murphy, $\ddagger \ddagger$ Mark B. Sandheinrich, $\S$ and James G. Wiener $\S$ \\ $\dagger$ Department of Biology and School of Environmental Studies, Queen's University, Kingston, Ontario, Canada \\ $\ddagger$ Department of Environmental Health Science, University of Michigan, Ann Arbor, Michigan, USA \\ $\S$ Ecotoxicology \& Wildlife Health Division, Environment Canada, Mount Pearl, Newfoundland and Labrador, Canada \\ $\|$ Biology Department, Hampden-Sydney College, Hampden-Sydney, Virginia, USA \\ \#National Institute for Scientific Research, Centre Eau Terre Environnement, Québec, Canada \\ $\dagger \dagger$ Department of Earth and Environmental Sciences, Wright State University, Dayton, Ohio, USA \\ †Department of Fisheries and Wildlife, Michigan State University, East Lansing, Michigan, USA \\ $\S \S$ River Studies Center, University of Wisconsin-La Crosse, La Crosse, Wisconsin, USA
}

(Submitted 12 October 2011; Returned for Revision 20 January 2012; Accepted 10 February 2012)

\begin{abstract}
Threshold concentrations associated with adverse effects of dietary exposure to methylmercury (MeHg) were derived from published results of laboratory studies on a variety of fish species. Adverse effects related to mortality were uncommon, whereas adverse effects related to growth occurred only at dietary $\mathrm{MeHg}$ concentrations exceeding $2.5 \mu \mathrm{g} \mathrm{g}^{-1}$ wet weight. Adverse effects on behavior of fish had a wide range of effective dietary concentrations, but generally occurred above $0.5 \mu \mathrm{g} \mathrm{g}^{-1}$ wet weight. In contrast, effects on reproduction and other subclinical endpoints occurred at dietary concentrations that were much lower $\left(<0.2 \mu \mathrm{gg}^{-1}\right.$ wet wt). Field studies generally lack information on dietary $\mathrm{MeHg}$ exposure, yet available data indicate that comparable adverse effects have been observed in wild fish in environments corresponding to high and low $\mathrm{MeHg}$ contamination of food webs and are in agreement with the threshold concentrations derived here from laboratory studies. These thresholds indicate that while differences in species sensitivity to $\mathrm{MeHg}$ exposure appear considerable, chronic dietary exposure to low concentrations of $\mathrm{MeHg}$ may have significant adverse effects on wild fish populations but remain little studied compared to concentrations in mammals or birds. Environ. Toxicol. Chem. 2012;31:15361547. (C) 2012 SETAC
\end{abstract}

Keywords_Fish Methylmercury Risk assessment Ecotoxicology

\section{INTRODUCTION}

Mercury $(\mathrm{Hg})$ is a widespread natural and anthropogenic contaminant of considerable ecological concern because it can be converted to methylmercury ( $\mathrm{MeHg}$ ), which biomagnifies through aquatic food webs and is highly toxic [1,2]. Obligate consumers of fish are considered to be most at risk for potential adverse effects of $\mathrm{MeHg}$ toxicity. Investigations of $\mathrm{MeHg}$ toxicity to aquatic organisms have traditionally focused on charismatic piscivorous mammals and birds because they represent the top trophic guild in many aquatic ecosystems (reviewed by Scheuhammer et al. [3]). Likewise, most consumption guidelines and/or criteria developed to mitigate the risks of $\mathrm{MeHg}$ toxicity are geared toward protection of human health and piscivorous wildlife $[3,4]$.

Numerous studies of wild fish in both contaminated and relatively pristine ecosystems have suggested that fish also are susceptible to adverse effects related to the ingestion of $\mathrm{MeHg}$ contaminated prey. These effects range from mortality [5] and emaciation [6] to sublethal effects such as oxidative stress [7-10], changes in gene transcription [11], and altered sex steroid levels [12]. Although fish are exposed to $\mathrm{MeHg}$ primarily through the diet [13], much scientific knowledge about the

All Supplemental Data may be found in the online version of this article.

* To whom correspondence may be addressed

(depewd@gmail.com).

Published online 30 April 2012 in Wiley Online Library

(wileyonlinelibrary.com). effects of $\mathrm{Hg}$ and $\mathrm{MeHg}$ on fish is derived from aqueous exposures to high concentrations of $\mathrm{MeHg}$ or dissolved $\mathrm{Hg}$ salts [14,15]. Moreover, aqueous exposures used in these studies were often orders of magnitude greater than concentrations in natural surface waters [16]. Such studies, although useful at the time, complicate interpretation of the observed adverse effects, particularly for risk-assessment purposes.

The toxicological effects of $\mathrm{MeHg}$ in freshwater fish were recently reviewed by Sandheinrich and Wiener [16], and we do not intend to revisit or replicate their comprehensive effort in the present study. Others have developed tissue-residue thresholds for adverse effects of $\mathrm{MeHg}$ in fish, using a combination of aqueous and dietary exposure data [17,18]. Tissue-residue approaches assume that a toxic effect is not observed unless the chemical reaches the site of action, and the target tissue is in equilibrium with external concentrations [19]. However, it is not clear that these assumptions are entirely appropriate for $\mathrm{MeHg}$ in fish because it is most often measured in dorsal muscle tissue and $\mathrm{MeHg}$ toxicity is not limited to one specific site of action. Moreover, tissue-residue thresholds developed to date $[17,18]$ do not distinguish between sublethal effects that may have dramatically different, yet highly meaningful ecological consequences. Therefore, an alternative and complementary approach to evaluate the risk posed to piscivorous fish based on exposure to $\mathrm{MeHg}$ is desirable. Our objective was to derive dietary threshold concentrations above which $\mathrm{MeHg}$ would be expected to elicit ecologically significant adverse effects in fish. 


\section{METHODS}

\section{Terminology}

We use the term "dietary exposure" to describe oral exposure that is in excess of, or unrelated to, nutritional requirements of the fish, because there are no known requirements for $\mathrm{Hg}$ or $\mathrm{MeHg}$ in living organisms [20]. A variety of experimental diets have been used to dose fish with $\mathrm{MeHg}$. Commercial diets containing protein, fatty acids, and minerals are typically used to simplify the feeding of cultured fish in experiments. Experimentally, $\mathrm{MeHg}$ is added, often as $\mathrm{MeHgCl}$, to these diets, which we refer to as commercial $\mathrm{MeHg}$ diets. Identification of specific dietary factors in nutritional research is typically conducted with specially formulated diets with purified ingredients. Although this type of diet was uncommon among the reviewed studies, we refer to these as purified or semipurified $\mathrm{MeHg}$ diets based on information in the source document. Live (or dead) prey items also were used in a few of the reviewed studies. Prey items can be contaminated naturally with $\mathrm{MeHg}$ in the environment or by injection of $\mathrm{MeHgCl}$ in a carrier solution; we refer to these diets as natural $\mathrm{MeHg}$ diets or prepared $\mathrm{MeHg}$ diets, respectively.

Uncertainty in the dietary $\mathrm{MeHg}$ exposures is introduced when investigators report nominal rather than measured concentrations in the experimental diet or when concentrations are reported on a dry-weight basis. In studies with prepared or natural $\mathrm{MeHg}$ diets, dietary concentrations generally have been reported on a wet-weight basis. In contrast, in experiments using commercial, purified, or semipurified $\mathrm{MeHg}$ diets, the moisture content of the diets was not reported and dietary concentrations were reported on a dry-weight basis. Fortunately, the moisture content of such diets appears to be low and relatively constant (on the order of 10\% [21]); and because most investigators either dried or lyophilized diets before use, we do not anticipate large variation in their moisture content. For comparative purposes, we have converted dry-weight concentrations to wet-weight equivalent concentrations using $75 \%$ water content to represent the typical moisture content of a small-prey fish [22]. Although this introduces additional uncertainty about the effective concentrations, this step ensured comparability to dietary concentrations from natural or prepared $\mathrm{MeHg}$ diets where moisture content may vary considerably.

\section{Literature search and screening criteria}

Dose-toxicity studies were identified in the literature with the Science Citation Index, SCOPUS indexing service, and ISI Web of Knowledge. Search terms included the following: "dietary," "methylmercury," "fish," and "oral exposure." A total of 87 studies were identified, but only 26 involved dietary exposure of fish to $\mathrm{MeHg}$. The others employed aqueous exposures or peritoneal injections of various $\mathrm{Hg}$ compounds (including $\mathrm{MeHg}$ ). Field studies were not included in the threshold derivation, due to uncertainty in estimating dietary exposure without complementary data. Studies were not used if either the experimental feeding or dosing regime was unclear or if concerns existed about the nature of the experimental controls. The studies that fell into these categories are discussed below on a case-by-case basis.

Among the 26 studies, six utilized marine or brackish water species (Supplemental References S1-S6). We initially planned to exclude marine species due to the relatively higher concentrations of selenium (Se) in marine organisms [23] and the potential confounding effects of Se on $\mathrm{MeHg}$ toxicity [24,25]. However, fish meal was the most likely source of protein in commercially prepared fish foods (commercial, semipurified, or purified $\mathrm{MeHg}$ diet) used in $88 \%$ of the studies with freshwater fishes. Commercial fish meal is often composed of marine forage fish (e.g., capelin, herring, anchovy), and levels of Se in these species can be high (1.2-4.3 $\mu \mathrm{g} \mathrm{g}^{-1}$ dry wt [26]). Levels of Se in Silver Cup Trout Food (Nelson and Sons), for example, which was used in several of the studies reviewed here, ranged from 0.6 to $1.2 \mu \mathrm{g} \mathrm{g}^{-1}$ dry weight [27]. Therefore, we could not assume that either commercial or purified $\mathrm{MeHg}$ diets for freshwater species had Se content any different from that of marine fish. The study by Pelletier and Audet (Supplemental Reference S1) was excluded due to concerns about the uncertainty of the dietary $\mathrm{MeHg}$ concentrations and the method of diet preparation. Two of the 20 studies with freshwater fish species (Supplemental References S2,S3) were excluded because they used wild-caught fish that had elevated body burdens of $\mathrm{MeHg}$ at the beginning of the experiment.

\section{Effect categorization and definition of adverse effects}

A variety of toxicological endpoints have been reported in the literature for fish, mammals, and birds exposed to $\mathrm{MeHg}$, but in a number of these studies, the ecological relevance of some endpoints at the individual or population level is unclear [3]. We classified adverse effects into categories that reflect or can be translated into ecologically meaningful population-level processes (Table 1). Either acute or chronic lethality (i.e., observed mortality) was ranked as the most severe outcome (severe risk in Table 1). Adverse effects that would have a clear impact on individuals (i.e., reduced growth rate or weight gain) or populations (i.e., reduced fecundity, spawning success, time spent spawning) were ranked as the next level of severity (i.e., high risk in Table 1). In some studies that reported reduced weight gain in dosed fish (Supplemental References S4, S5 [ad libitum group], S6), reduced food consumption was also documented and complicates interpretation. For these studies, we included a growth-related end point only if evidence for growth-rate or weight-gain impairment could be separated from effects of food consumption if reported. For example, Webber and Haines (Supplemental Reference S6) noted reduced weight gain and food consumption in golden shiner (Notemigonus crysoleucas) fed $0.455 \mu \mathrm{gg}^{-1} \mathrm{MeHg}$ wet weight but not in those fed $0.959 \mu \mathrm{g} \mathrm{g}^{-1} \mathrm{MeHg}$ wet weight. We therefore did not attribute reductions in weight gain in the $0.455-\mu \mathrm{g} \mathrm{g}^{-1}$ treatment group to $\mathrm{MeHg}$ in the diet. Sublethal effects related to behavioral, biochemical, histological, or genomic effects were considered to be indicators of adverse effects; but the relevance to individuals or population-level effects is not well understood. These effects were ranked as the next level of severity (moderate risk, Table 1). Due to the diversity in endpoints measured in this category and the small number of species examined, it was not possible to construct dose-response relationships among studies for a common endpoint (e.g., lipid peroxidation in organ tissue).

\section{Threshold derivation}

We determined the no observed adverse effect level (NOAEL) and the lowest observed adverse effect level (LOAEL) dietary concentrations from statistics reported in the source documents. Unbounded NOAEL (i.e., no observed adverse effect up to the highest diet tested) was included as a conservative estimate of true NOAEL. Two challenges arose while summarizing these effect levels. The first was how to deal with either multiple endpoints from the same study or the same 
Table 1. Relative severity and classification of toxicological end points in fish exposed to dietary $\mathrm{MeHg}$

\begin{tabular}{lll}
\hline Effect severity rating & \multicolumn{1}{c}{ Endpoint classification } & \multicolumn{1}{c}{ Endpoints considered $^{\mathrm{a}}$} \\
\hline $\begin{array}{l}\text { Severe } \\
\text { High }\end{array}$ & Severe adverse effects & Acute or chronic lethality (1) \\
& Adverse effects on growth and development or & Reduced growth (weight or length) (2a) \\
& reproductive success & Emaciation (2a) \\
& & Reduced spawning success (2b) \\
& & Reduced fecundity (2b) \\
& & Gonadosomatic index (2b) \\
& & Altered spawning behavior (2b) \\
Moderate & Adverse effects with unknown or poorly characterized steroids (2b) & Altered blood/plasma biochemistry (3a) \\
& ecological consequences & Altered neurochemistry (3a) \\
& & Changes in gene transcription (3a) \\
& Changes in cellular physiology (3a) \\
& Pathological damage to organs or tissues (3a) \\
& Altered behavior (e.g., predator evasion, impaired gross motor \\
\end{tabular}

${ }^{a}$ Numbers in parentheses indicate groupings for end points in Table 2.

endpoint in multiple studies with the same fish species. The second was how to account for interspecies variation in sensitivity or susceptibility to $\mathrm{MeHg}$ toxicity. To address the first issue, we selected the most sensitive endpoint value when there were multiple effects measured from the same adverse-effect category. For example, Wobeser (Supplemental Reference S7) observed deformation of the gill epithelium in rainbow trout (Oncorhynchus mykiss) at dietary $\mathrm{MeHg}$ concentrations below those where internal lesions and increased blood cell volume were observed. Because both of these effects fall into the "moderate" adverse-effect classification (i.e., effects with unclear ecological relevance), we used the most sensitive endpoint to define the LOAEL. For studies that used the same species and similar endpoints (e.g., reproductive success in fathead minnows, Pimephales promelas) (Supplemental References S8-S10), variation between experimental protocols or the fish used may introduce additional variation in endpoint sensitivity, but inclusion of the NOAEL and LOAEL from each study would weight the results more heavily toward this species. For these species and suitable endpoints, we calculated the geometric mean of the NOAEL and/or LOAEL across studies.

To address the second issue, species sensitivity distributions are typically used in combination with probabilistic models to estimate the 5th percentile of the NOAEL distribution, to establish a protective threshold for approximately $95 \%$ of the species examined [17]. While such approaches are indeed valuable, they can be overly conservative if the number of species is small (fewer than 20 [28]) due to extrapolation beyond the lowest NOAEL [17,28]. Moreover, these approaches are best suited to standardized laboratory test procedures, where variation among experimental design and other factors is minimized.

For this review, we calculated estimated protective dietary thresholds following two approaches advocated by Beckvar et al. [17]. The first approach ranks the NOAEL, unbounded NOAEL, and LOAEL in order of magnitude; the highest NOAEL (or unbounded NOAEL) below the lowest LOAEL is selected as the protective threshold. The second follows the modification of the approach used by the Canadian Council of Ministers of Environment (CCME) for sediment-toxicity thresholds [29] and is superior to the 5th-percentile approach when analyzing small data sets [17]. The threshold effect level is estimated from the 50th percentile of the NOAEL and the
15 th percentile of the LOAEL with the equation

$$
\mathrm{TEL}=\sqrt{\text { NOAEL }_{50} x \mathrm{LOAEL}_{15}}
$$

For consistency, we selected the lowest concentration (calculated threshold effect level or ranked NOAEL) as the protective threshold value unless otherwise indicated.

\section{RESULTS AND DISCUSSION}

Twenty experimental studies were deemed suitable for this analysis based on our screening criteria. Toxicological results from twelve fish species and experiments ranging from 25 to $600 \mathrm{~d}$ are summarized in Table 2. Most studies employed single cohorts of either juvenile or adult fish, although three incorporated transgenerational effects or assessed impacts on offspring (Supplemental References S8,S11,S12). Despite the limited number of studies, a substantial array of endpoints was examined (Table 2). We summarize the compiled data as cumulative frequency distributions in a manner similar to that used to construct species sensitivity distributions (Figs. 1-3). Although several species were not used to calculate the threshold concentrations, we show data for them on the graphics for completeness where possible.

Clear differences in sensitivity are apparent among endpoints in the high- and moderate-risk categories. Effects on reproduction occurred at dietary concentrations far lower than those associated with negative impacts on growth (Fig. 2), and biochemical, histological, and genotoxic endpoints were associated with dietary concentrations lower than those associated with behavioral endpoints (Fig. 3). For calculation of thresholds related to these groupings of endpoints (as described in the Methods section), we a posteriori subdivided these two classes according to the apparent ranges in sensitivity and endpoint category (i.e., reproduction and growth; histological, biochemical, and genomic; behavioral).

\section{Severe ecological risk: Lethality}

Acute or chronic mortality of fish was readily determined from each study. Mortality was attributed to dietary exposure of $\mathrm{MeHg}$, although Scherer et al. (Supplemental Reference S13) observed substantial mortality of adult walleye (Sander vitreus, $\sim 27 \%$ ) in the low-dose group (control) in early stages of their study (attributed to cleaning product residues), but this was far exceeded by mortality in the high-dose treatment group $(88 \%)$. 
Table 2. Summary of test conditions and effects of dietary $\mathrm{MeHg}$ exposure on different fish species as compiled from the literature

\begin{tabular}{|c|c|c|c|c|c|c|c|c|c|c|}
\hline Species (family) & Age & Diet type & $\begin{array}{l}\text { Dose } \\
\text { level }\end{array}$ & $\begin{array}{l}\text { Water } \\
\text { temperature } \\
\left({ }^{\circ} \mathrm{C}\right)\end{array}$ & $\begin{array}{l}\text { Daily } \\
\text { ration }\end{array}$ & $\begin{array}{l}\text { End point } \\
\text { (category) }\end{array}$ & $\begin{array}{l}\text { Duration } \\
\text { (d) }\end{array}$ & $\begin{array}{l}\text { NOAEL } \\
\left(\mu g^{-1}\right)^{a}\end{array}$ & $\begin{array}{l}\text { LOAEL } \\
\left(\mu \mathrm{gg}^{-1}\right)\end{array}$ & Ref \\
\hline \multicolumn{11}{|l|}{$\begin{array}{l}\text { Freshwater } \\
\text { (Percidae) }\end{array}$} \\
\hline \multirow[t]{3}{*}{ Walleye } & \multirow[t]{3}{*}{ A } & \multirow[t]{3}{*}{ Natural $^{\mathrm{c}}$} & \multirow[t]{3}{*}{2} & \multirow[t]{3}{*}{ NA } & \multirow{3}{*}{$\begin{array}{c}\text { Ad lib. } \\
\left(2 \mathrm{~g} \mathrm{fish}^{-1}\right)\end{array}$} & Mortality (1) & \multirow[t]{3}{*}{$>276$} & $>0.41$ & 7.92 & $\mathrm{~S} 13$ \\
\hline & & & & & & Emaciation (2a) & & $>0.41$ & 7.92 & S13 \\
\hline & & & & & & $\downarrow$ Response to light (3b) & & $>0.41$ & 7.92 & S13 \\
\hline \multirow[t]{4}{*}{ Walleye } & \multirow[t]{4}{*}{$\mathrm{J}$} & \multirow{4}{*}{$\begin{array}{c}\mathrm{MeHgCl} \\
\text { (prepared) }^{\mathrm{d}}\end{array}$} & \multirow[t]{4}{*}{3} & \multirow[t]{4}{*}{20} & $1-1.5 \mathrm{~g} \mathrm{fish}^{-1}$ & Mortality (1) & 180 & $>0.987$ & & S14 \\
\hline & & & & & & $\downarrow$ Weight gain (2a) $\widehat{\jmath}$ & & $>0.137$ & 0.987 & S14 \\
\hline & & & & & & $\downarrow$ GSI $(2 b)$ & & $>0.04$ & 0.137 & S14 \\
\hline & & & & & & $\downarrow$ Plasma cortisol (3a) & & $>0.04$ & 0.137 & $\mathrm{~S} 14$ \\
\hline (Salmonidae) & & & & & & & & & & \\
\hline Atlantic salmon & $\mathrm{J}$ & $\mathrm{MeHgCl}$ & 3 & $9-11$ & $1.6 \%$ & Mortality (1) & 120 & $>2.12^{\mathrm{f}}$ & & S19 \\
\hline & & (purified) $^{\mathrm{e}}$ & & & & $\begin{array}{l}\text { Abnormal brain } \\
\text { pathology }(3 a)\end{array}$ & & $>0.075^{\mathrm{f}}$ & $1.09^{\mathrm{f}}$ & S19 \\
\hline & & & & & & Altered brain & & $>0.075^{\mathrm{f}}$ & $1.09^{\mathrm{f}}$ & S19 \\
\hline & & & & & & biochemistry (3a) & & & & \\
\hline & & & & & & $\begin{array}{l}\downarrow \text { Postfeeding } \\
\text { behavior }(3 \mathrm{~b})\end{array}$ & & $1.09^{f}$ & $2.12^{\mathrm{f}}$ & $\mathrm{S} 19$ \\
\hline Atlantic salmon & $\mathrm{J}$ & $\mathrm{MeHgCl}$ & 4 & $9-11$ & $2-2.6 \%$ & Mortality (1) & 120 & $>2.12^{\mathrm{f}}$ & & S20 \\
\hline & & 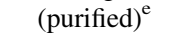 & & & & $\downarrow$ Weight $(2 \mathrm{a})$ & & $>2.12^{\mathrm{f}}$ & & S20 \\
\hline & & & & & & $\begin{array}{c}\uparrow \text { Intestinal } \\
\text { pathology (3a) }\end{array}$ & & $0.03^{\mathrm{f}}$ & $0.158^{\mathrm{f}}$ & $\mathrm{S} 20$ \\
\hline & & & & & & $\uparrow$ Hematocrit (3a) & & $0.03^{f}$ & $0.158^{f}$ & $\mathrm{~S} 20$ \\
\hline Rainbow trout & $\mathrm{J}$ & $\mathrm{MeHgCl}$ & 4 & $10.5 \pm 1.5$ & Ad lib. & Mortality (1) & 84 & $>23.7^{\mathrm{f}}$ & & S5 \\
\hline & & $(\text { commercial })^{\mathrm{g}}$ & & & & $\uparrow$ Lethargy (3b) & & $11.73^{f}$ & $23.7^{\mathrm{f}}$ & S5 \\
\hline & & & & & & Discoloration (3a) & & $11.73^{\mathrm{f}}$ & $23.7^{\mathrm{f}}$ & S5 \\
\hline Rainbow trout & $\mathrm{J}$ & $\mathrm{MeHgCl}$ & 3 & $10.5 \pm 1.5$ & $2 \%$ & Mortality (1) & 84 & $>19.13^{\mathrm{f}}$ & & S5 \\
\hline & & $(\text { commercial })^{\mathrm{h}}$ & & & & $\downarrow$ Weight gain (2a) & & $>0.025^{\mathrm{f}}$ & $5.8^{\mathrm{f}}$ & S5 \\
\hline & & & & & & $\uparrow$ Lethargy (3b) & & $5.8^{\mathrm{f}}$ & $19.13^{\mathrm{f}}$ & S5 \\
\hline & & & & & & Discoloration (3a) & & $5.8^{\mathrm{f}}$ & $19.13^{\mathrm{f}}$ & S5 \\
\hline Rainbow trout & $\mathrm{J}$ & $\mathrm{MeHgCl}$ & 5 & $10 \pm 0.7$ & $3-4 \%$ & Mortality (1) & 105 & $>24^{\mathrm{i}}$ & & S7 \\
\hline & & $(\text { commercial })^{\mathrm{h}}$ & & & & $\downarrow$ Weight (2a) & & $8^{\mathrm{i}}$ & $16^{\mathrm{i}}$ & S7 \\
\hline & & & & & & $\begin{array}{c}\text { Deformation of gill } \\
\text { epithelium (3a) }\end{array}$ & & $>0.05^{\mathrm{i}}$ & $4^{i}$ & S7 \\
\hline & & & & & & $\begin{array}{l}\uparrow \text { Packed cell volume } \\
\text { (blood) (3a) }\end{array}$ & & $8^{\mathrm{i}}$ & $16^{\mathrm{i}}$ & S7 \\
\hline & & & & & & Internal lesions (3a) & & $>24^{\mathrm{i}}$ & & S7 \\
\hline & & & & & & $\begin{array}{l}\text { Altered resting } \\
\text { position }(3 b)\end{array}$ & & $8^{i}$ & $16^{\mathrm{i}}$ & S7 \\
\hline & & & & & & Discoloration (3a) & & $16^{\mathrm{i}}$ & $24^{\mathrm{i}}$ & S7 \\
\hline Rainbow trout ${ }^{\mathrm{b}}$ & $\mathrm{J}$ & $\mathrm{MeHgCl}$ & 5 & 16 & $\sim 5 \%$ & Mortality (1) & $84-280$ & $5^{\mathrm{i}}$ & $25^{\mathrm{i}}$ & S16 \\
\hline & & $(\text { commercial })^{\mathrm{j}}$ & & & & $\downarrow$ Weight gain $(2 \mathrm{a})$ & & $>0^{\mathrm{i}}$ & $1^{\mathrm{i}}$ & S16 \\
\hline & & & & & & $\begin{array}{l}\text { Impaired locomotion } \\
\text { and feeding }(3 b)\end{array}$ & & $5^{i}$ & $25^{\mathrm{i}}$ & S16 \\
\hline & & & & & & Discoloration (3a) & & $5^{\mathrm{i}}$ & $25^{\mathrm{i}}$ & S16 \\
\hline & & & & & & CNS pathology (3a) & & $5^{\mathrm{i}}$ & $25^{\mathrm{i}}$ & S16 \\
\hline (Cyprinidae) & & & & & & & & & & \\
\hline Fathead minnow & $\mathrm{J}-\mathrm{A}$ & $\mathrm{MeHgCl}$ & 3 & $23.6 \pm 0.1$ & $5 \%$ & Mortality (1) & 250 & $>0.983^{\mathrm{f}}$ & & S9 \\
\hline & & $(\text { commercial })^{\mathrm{k}}$ & & & & $\downarrow$ GSI (2b) & & $>0.015^{\mathrm{f}}$ & $0.218^{\mathrm{f}}$ & $\mathrm{S} 18$ \\
\hline & & & & & & $\downarrow$ Spawning success $(2 b)$ & & $>0.015^{\mathrm{f}}$ & $0.218^{\mathrm{f}}$ & S18 \\
\hline & & & & & & $\uparrow$ Apoptosis in ovaries $(2 \mathrm{~b})$ & & $>0.015^{\mathrm{f}}$ & $0.218^{\mathrm{f}}$ & S18 \\
\hline & & & & & & $\downarrow$ Sex steroids $(2 \mathrm{~b})$ & & $>0.015^{\mathrm{f}}$ & $0.218^{\mathrm{f}}$ & S18 \\
\hline Fathead minnow & $\mathrm{J}-\mathrm{A}$ & $\mathrm{MeHgCl}$ & 4 & $25 \pm 1$ & $5 \%$ & Mortality (1) & 195 & $>2.12^{\mathrm{f}}$ & & S8 \\
\hline & & $(\text { commercial })^{\mathrm{k}}$ & & & & $\downarrow$ GSI (2b) & & $>0.015^{\mathrm{f}}$ & $0.22^{\mathrm{f}}$ & S8 \\
\hline & & & & & & $\downarrow$ Spawning success $(2 b)$ & & $>0.015^{\mathrm{f}}$ & $0.22^{\mathrm{f}}$ & $\mathrm{S} 8$ \\
\hline Fathead minnow & $\mathrm{J}-\mathrm{A}$ & $\mathrm{MeHgCl}$ & 3 & $25 \pm 1$ & $5 \%$ & Mortality (1) & NA & $>0.983^{\mathrm{f}}$ & & S10 \\
\hline & & $(\text { commercial })^{\mathrm{k}}$ & & & & $\downarrow$ Spawning behavior $(2 \mathrm{~b})$ & & $>0.015^{\mathrm{f}}$ & $0.218^{f}$ & $\mathrm{~S} 10$ \\
\hline & & & & & & $\downarrow$ Spawning success $(2 b)$ & & $>0.015^{\mathrm{f}}$ & $0.218^{\mathrm{f}}$ & S10 \\
\hline & & & & & & $\downarrow$ Testosterone $(2 \mathrm{~b})$ & & $>0.015^{\mathrm{f}}$ & $0.218^{\mathrm{f}}$ & S10 \\
\hline Fathead minnow & $\mathrm{J}-\mathrm{A}$ & $\mathrm{MeHgCl}$ & 3 & $23.6 \pm 0.1$ & $5 \%$ & Mortality (1) & 600 & $>0.983^{\mathrm{f}}$ & & S24 \\
\hline & & $(\text { commercial })^{\mathrm{k}}$ & & & & \pm Gene transcription $(3 a)$ & & $>0.015^{\mathrm{f}}$ & $0.218^{\mathrm{f}}$ & S24 \\
\hline Golden shiner & A & $\mathrm{MeHgCl}$ & 3 & 23 & $2 \%$ & Mortality (1) & 90 & $>0.959$ & & S6 \\
\hline & & ${\text { (semipurified })^{1}}^{1}$ & & & & $\downarrow$ Weight gain $(2 \mathrm{a})^{\mathrm{m}}$ & & $>0.959$ & & S6 \\
\hline & & & & & & Brain biochemistry (3a) & & $>0.959$ & & S6 \\
\hline & & & & & & $\begin{array}{c}\uparrow \text { Shoal dispersal } \\
\text { and settling } \\
\text { time }(3 b)\end{array}$ & & 0.455 & 0.959 & S6 \\
\hline Sacramento & $\mathrm{J}$ & $\mathrm{MeHgCl}$ & 4 & $23 \pm 1$ & $1-1.3 \%$ & Mortality (1) & $70-247$ & $5.53^{\mathrm{f}}$ & $13.78^{f}$ & $\mathrm{~S} 17$ \\
\hline blackfish & & $(\text { commercial })^{\mathrm{n}}$ & & & & $\downarrow$ Growth rate $(2 \mathrm{a})$ & & $5.53^{\mathrm{f}}$ & $13.78^{\mathrm{f}}$ & S17 \\
\hline & & & & & & Discoloration (3a) & & $>13.78^{\mathrm{f}}$ & & S17 \\
\hline & & & & & & $\mathrm{O}_{2}$ consumption $(3 \mathrm{a})$ & & $>13.78^{\mathrm{f}}$ & & S17 \\
\hline
\end{tabular}


Table 2. (Continued)

\begin{tabular}{|c|c|c|c|c|c|c|c|c|c|c|}
\hline Species (family) & Age & Diet type & $\begin{array}{l}\text { Dose } \\
\text { level }\end{array}$ & $\begin{array}{c}\text { Water } \\
\text { temperature } \\
\left({ }^{\circ} \mathrm{C}\right)\end{array}$ & $\begin{array}{l}\text { Daily } \\
\text { ration }\end{array}$ & $\begin{array}{l}\text { End point } \\
\text { (category) }\end{array}$ & $\begin{array}{l}\text { Duration } \\
\text { (d) }\end{array}$ & $\begin{array}{l}\text { NOAEL } \\
\left(\mu \mathrm{gg}^{-1}\right)^{\mathrm{a}}\end{array}$ & $\begin{array}{l}\text { LOAEL } \\
\left(\mu \mathrm{gg}^{-1}\right)\end{array}$ & $\mathrm{Re}$ \\
\hline Zebrafish & A $(\hat{o})$ & $\begin{array}{c}\mathrm{MeHgCl} \\
(\text { commercial) }\end{array}$ & 2 & $24 \pm 0.5$ & $5 \%$ & $\begin{array}{l}\text { Mortality (1) } \\
\downarrow \text { Mitochondrial } \\
\text { metabolism (3a) }\end{array}$ & 49 & $\begin{array}{l}>3.38^{\mathrm{f}} \\
>0.02^{\mathrm{f}}\end{array}$ & $3.38^{\mathrm{f}}$ & $\begin{array}{l}\text { S25 } \\
\text { S25 }\end{array}$ \\
\hline Zebrafish & $\mathrm{A}(\widehat{\jmath})$ & $\begin{array}{c}\mathrm{MeHgCl} \\
\text { (commercial) }^{\mathrm{o}}\end{array}$ & 2 & $24 \pm 0.5$ & $5 \%$ & $\begin{array}{c}\text { Mortality (1) } \\
\pm \text { Gene transcription (3a) }\end{array}$ & 25 & $\begin{array}{l}>3.38^{\mathrm{f}} \\
>0.02^{\mathrm{f}}\end{array}$ & $3.38^{\mathrm{f}}$ & $\begin{array}{l}\text { S23 } \\
\text { S23 }\end{array}$ \\
\hline Zebrafish & $\mathrm{A}(\widehat{\jmath})$ & $\begin{array}{c}\mathrm{MeHgCl} \\
\text { (commercial) }^{\circ}\end{array}$ & 3 & $24 \pm 0.5$ & $5 \%$ & $\begin{array}{l}\text { Mortality (1) } \\
\uparrow \text { SOD gene induction in } \\
\text { muscle (3a) }\end{array}$ & 63 & $\begin{array}{l}>3.38^{\mathrm{f}} \\
>0.02^{\mathrm{f}}\end{array}$ & $0.125^{\mathrm{f}}$ & $\begin{array}{l}\text { S22 } \\
\text { S22 }\end{array}$ \\
\hline Zebrafish & A & 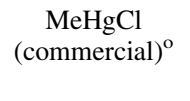 & 2 & $24 \pm 0.5$ & $5 \%$ & $\begin{array}{l}\text { Mortality (1) } \\
\uparrow \text { Mitochondrial } \\
\text { pathology (3a) }\end{array}$ & 63 & $\begin{array}{l}>3.38^{\mathrm{f}} \\
>0.02^{\mathrm{f}}\end{array}$ & $3.38^{\mathrm{f}}$ & $\begin{array}{l}\mathrm{S} 21 \\
\mathrm{~S} 21\end{array}$ \\
\hline $\begin{array}{l}\text { Marine/Brackish } \\
\text { (Sciaenidae) }\end{array}$ & & & & & & & & & & \\
\hline $\begin{array}{l}\text { Atlantic croaker } \\
\text { (Fundulidae) }\end{array}$ & A & Natural $^{\mathrm{p}}$ & 3 & 22 & Ad lib. & $\begin{array}{l}\text { Mortality (1) } \\
\text { Altered larval behavior (2b) }\end{array}$ & 30 & $\begin{array}{l}>0.1 \\
>0\end{array}$ & 0.05 & $\begin{array}{l}\text { S12 } \\
\text { S12 }\end{array}$ \\
\hline Killifish & A & $\begin{array}{c}\mathrm{MeHgCl} \\
\left(_{(\text {commercial })^{\mathrm{q}}}\right.\end{array}$ & 5 & 22 & Ad lib. & $\begin{array}{c}\mathrm{F}_{0} \text { Mortality }(1) \\
\mathrm{F}_{0} \text { Growth }(2 \mathrm{a}) \\
\downarrow \mathrm{F}_{1} \text { fecundity }(2 \mathrm{~b}) \\
\mathrm{F}_{1} \text { Offspring sex ratio }(2 \mathrm{~b}) \\
\downarrow \mathrm{F}_{1} \text { Fertilization success }(2 \mathrm{~b}) \\
\mathrm{F}_{0} \text { Behavior }(3 \mathrm{a})\end{array}$ & 42 & $\begin{array}{c}0.5 \\
>54 \\
>0.07 \\
1.9 \\
5.6 \\
0.5\end{array}$ & $\begin{array}{l}1.9(\hat{)}) \\
\\
0.5 \\
5.6 \\
54 \\
1.9(\hat{o})\end{array}$ & $\begin{array}{l}\text { S11 } \\
\text { S11 } \\
\text { S11 } \\
\text { S11 } \\
\text { S11 } \\
\text { S11 }\end{array}$ \\
\hline $\begin{array}{l}\text { (Acipenseridae) } \\
\text { Beluga sturgeon }\end{array}$ & $\mathrm{J}$ & $\begin{array}{c}\mathrm{MeHgCl} \\
\text { (semipurified) }\end{array}$ & 4 & 25 & NA & $\begin{array}{c}\text { Mortality (1) } \\
\downarrow \text { Growth rate }(2 \mathrm{a})^{\mathrm{s}} \\
\text { Discoloration (3b) }\end{array}$ & 70 & $\begin{array}{l}1.97^{\mathrm{f}} \\
0.19^{\mathrm{f}} \\
1.97^{\mathrm{f}}\end{array}$ & $\begin{array}{l}4.05^{\mathrm{f}} \\
1.97^{\mathrm{f}} \\
4.05^{\mathrm{f}}\end{array}$ & $\begin{array}{l}\text { S4 } \\
\text { S4 }\end{array}$ \\
\hline Beluga sturgeon & $\mathrm{J}$ & $\begin{array}{c}\mathrm{MeHgCl} \\
\text { (semipurified) }^{\mathrm{r}}\end{array}$ & 4 & 25 & NA & $\begin{array}{l}\uparrow \text { Blood metabolites and } \\
\text { enzyme activity (3b) }\end{array}$ & 32 & $>0.01^{\mathrm{f}}$ & $0.19^{\mathrm{f}}$ & S26 \\
\hline Green sturgeon & $\mathrm{J}$ & $\begin{array}{c}\mathrm{MeHgCl} \\
\text { (semipurified) }\end{array}$ & 4 & 18 & $2-3 \%$ & $\begin{array}{c}\text { Mortality (1) } \\
\downarrow \text { Growth rate (2a) } \\
\downarrow \text { HSI (2a) }\end{array}$ & 56 & $\begin{array}{l}5.13^{\mathrm{f}, \mathrm{u}} \\
5.13^{\mathrm{f}, \mathrm{u}} \\
9.73^{\mathrm{f}, \mathrm{u}}\end{array}$ & $\begin{array}{l}9.73^{\mathrm{f}, \mathrm{u}} \\
9.73^{\mathrm{f}, \mathrm{u}} \\
24.3^{\mathrm{f}, \mathrm{u}}\end{array}$ & $\begin{array}{l}\text { S15 } \\
\text { S15 } \\
\text { S15 }\end{array}$ \\
\hline White sturgeon & $\mathrm{J}$ & $\begin{array}{c}\mathrm{MeHgCl} \\
\text { (semipurified) }^{\mathrm{t}}\end{array}$ & 4 & 18 & $2-3 \%$ & $\begin{array}{c}\text { Mortality (1) } \\
\downarrow \text { Growth rate (2a) }\end{array}$ & 56 & $\begin{array}{l}9.73^{\mathrm{f}, \mathrm{u}} \\
9.73^{\mathrm{f,u}}\end{array}$ & $\begin{array}{l}24.3^{\mathrm{f}, \mathrm{u}} \\
24.3^{\mathrm{f,u}}\end{array}$ & $\begin{array}{l}\text { S15 } \\
\text { S15 }\end{array}$ \\
\hline
\end{tabular}

${ }^{\mathrm{a}}$ Unbounded NOAEL is denoted by $>$.

${ }^{\mathrm{b}}$ Only details of the experimental feeding with laboratory-prepared food are included here.

${ }^{\mathrm{c}}$ Shredded northern pike from Clay Lake, Ontario, Canada.

${ }^{\mathrm{d}} \mathrm{MeHgCl}$ injected into catfish fillets and fathead minnows.

${ }^{\mathrm{e}}$ Described fully in Berntssen et al. (Supplemental References S19,S20).

${ }^{\mathrm{f}}$ Conversion from dry weight to wet weight equivalent using $75 \%$ moisture content.

${ }^{g}$ Trout chow (Martin's Feed Mills).

${ }^{\mathrm{h}}$ 5:1 mixture of pork liver and commercial trout feed, nominal concentrations (G. Wobeser, University of Saskatchewan, Saskatoon, SK, Canada, personal communication).

${ }^{i}$ Presented as nominal (wet wt) rather than assayed concentrations.

${ }^{\mathrm{j}}$ Commercial Trout Chow (unknown origin).

${ }^{\mathrm{k}}$ Starter soft moist fish food (Nelson and Sons).

${ }^{1}$ Lochmann and Phillips (supplemental Reference S27).

${ }^{\mathrm{m}}$ Reduced growth rate due to reduced food consumption.

${ }^{\mathrm{n}}$ Sterling Silver Cup Fish food (Nelson and Sons Inc.).

${ }^{\circ}$ Dr. Bassleer biofish flakes.

${ }^{\mathrm{p}}$ Shrimp (control diet), blue marlin (low diet), blue marlin and contaminated shrimp (high diet).

${ }^{\mathrm{q}}$ Nutra Fry fish food (Moore-Clark).

${ }^{\mathrm{r}}$ See reference for details.

${ }^{\mathrm{s}}$ Reduced growth rate measured during time period $1(0-35 \mathrm{~d})$ when food-consumption rates were constant.

${ }^{\mathrm{t}}$ See reference for details.

${ }^{\mathrm{u}}$ Concentrations reported as assayed amounts (S.S.O. Hung, University of California-Davis, Davis, CA, USA, personal communication).

Species $=$ test species used in experiment; age during exposure $=$ adult $(\mathrm{A})$, juvenile $(\mathrm{J})$, or juvenile to adult $(\mathrm{J}-\mathrm{A})$; dose levels $=$ number of MeHg exposure levels used (including controls); daily ration = mass of food provided per fish or percent of body weight per day; end points = end points measured (category of severity); duration $=$ days of experimental exposure; $\mathrm{NOAEL}=$ no observed adverse effect level in micrograms per gram (wet wt); LOAEL $=$ lowest observed adverse effect level in micrograms per gram (wet wt); GSI = gonadosomatic index, HSI = hepatosomic index.

Friedmann et al. (Supplemental Reference S14) also noted some early mortality of juvenile walleye in both control and treatment groups. This may reflect the difficulty of maintaining walleyes in laboratory conditions. Lee et al. (Supplemental Reference S15) observed significant mortality in both juvenile green (Acipenser medirostris) and white (Acipenser transmontanus) sturgeon, and Gharaei et al. (Supplemental Reference S4) observed $100 \%$ mortality of juvenile beluga sturgeon (Huso huso) at the highest dose tested. In general, mortality was uncommon, was observed only in studies that exceeded $49 \mathrm{~d}$ in duration, and occurred in a dose- and time-dependent manner (Table 2) (Supplemental References S4,S13,S15-S17). The frequency distribution of NOAELs and LOAELs for mortality illustrates that lethal effects occurred over a range of 


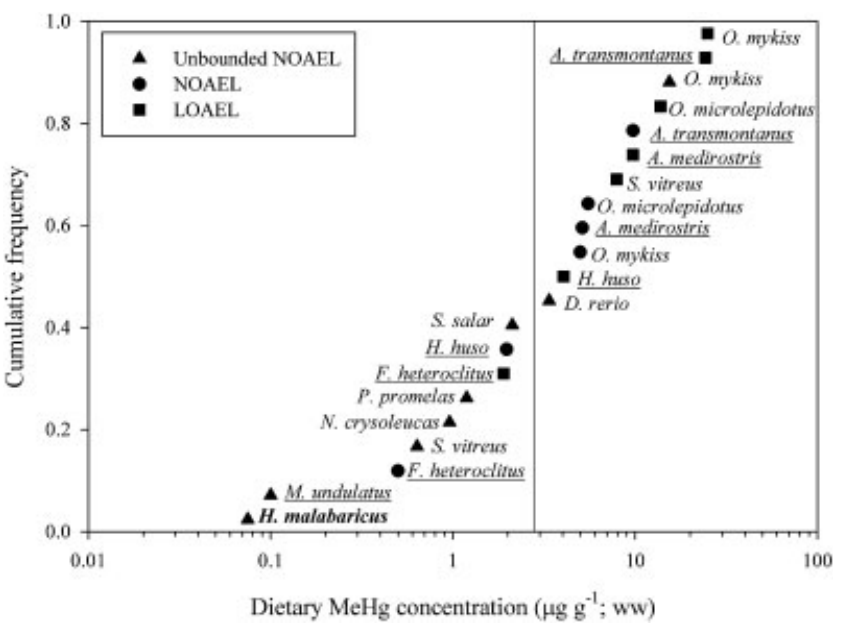

Fig. 1. Cumulative frequency distribution of unbounded no observed adverse effect levels (NOAELs), NOAELs, and lowest observed adverse effect levels (LOAELs) for mortality endpoint. Species are as follows: Atlantic croaker (Micropogonias undulatus), Atlantic salmon (Salmo salar), beluga sturgeon (Huso huso), fathead minnow (Pimephales promelas), green sturgeon (Acipenser medirostris), golden shiner (Notemigonous crysoleucas), killifish (Fundulus heteroclitus), rainbow trout (Oncorhynchus mykiss), Sacramento blackfish (Orthodon microlepidotus), trahira (Hoplias malabaricus), walleye (Sander vitreus), white sturgeon (Acipenser transmontanus), and zebrafish (Danio rerio). Species with bolded names are shown for comparison but were not used in derivation of thresholds (see Methods section). Species with underlined names are marine or brackish water species. Solid line indicates recommended protective threshold for lethality. Note that Atlantic salmon (S. salar) experiments were conducted with hatchery-reared fish in freshwater.

concentrations but did not occur unless dietary concentrations were $1.9 \mu \mathrm{gg}^{-1}$ wet weight or greater (Fig. 1).

The ranking approach and calculation of a threshold effect level gave threshold values of 1.2 and $2.8 \mu \mathrm{g} \mathrm{g}^{-1}$ wet weight, respectively, for this end point (Table 3 ). The ranked NOAEL of $1.2 \mu \mathrm{g} \mathrm{g}^{-1}$ wet weight is probably overly conservative because it is lower than the ranked NOAEL for growth (see below).

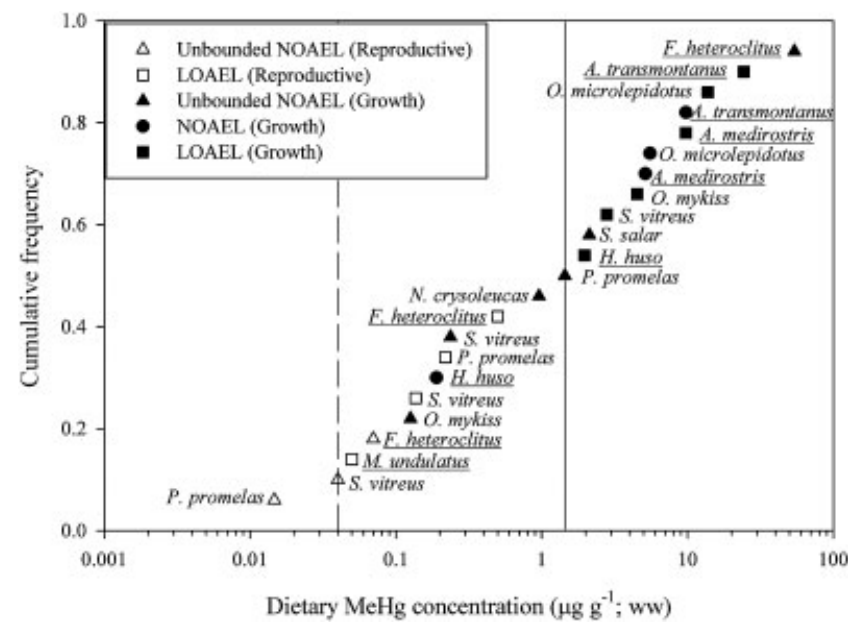

Fig. 2. Cumulative frequency distribution of unbounded no observed adverse effect levels (NOAELs), NOAELs, and lowest observed adverse effect levels (LOAELs) for reproductive (open symbols) and growth-related (filled symbols) endpoints. For species names, see Fig. 1. Species with underlined names are marine or brackish water species. Solid and dashed lines represent recommended protective thresholds for growth and reproductive effects, respectively. Note that Atlantic salmon ( $S$. salar) experiments were conducted with hatchery-reared fish in freshwater.

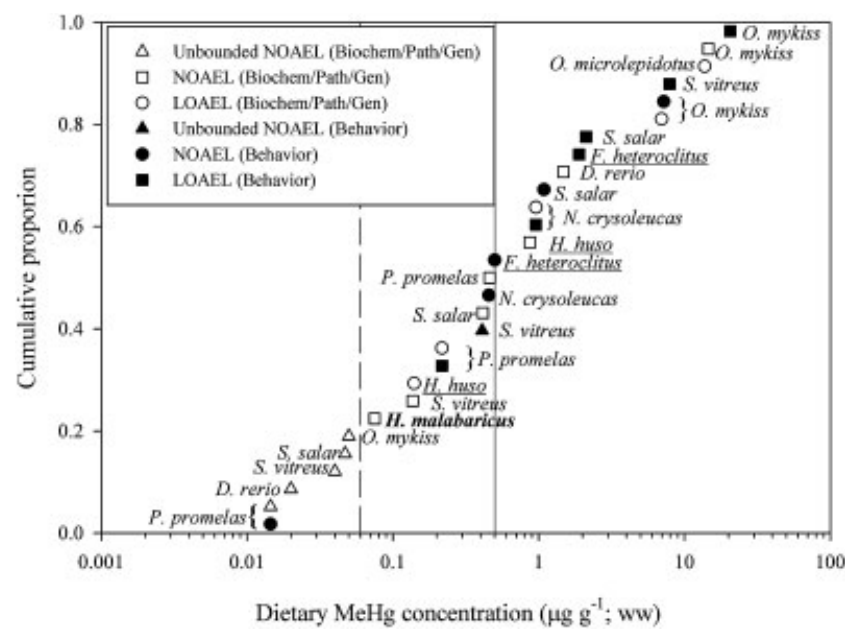

Fig. 3. Cumulative frequency distribution of unbounded no observed adverse effect levels (NOAELs), NOAELs, and lowest observed adverse effect levels (LOAELs) for biochemical, histopathological, genotoxic (open symbols) and behavioral (filled symbols) endpoints. For species names, see Fig. 1. Species with bolded names are shown for comparison but were not used in derivation of thresholds (see Methods section). Species with underlined names are marine or brackish water species. Solid and dashed vertical lines represent recommended protective thresholds for behavioral and for biochemical, histological, and genomic effects, respectively. Note that Atlantic salmon ( $S$. salar) experiments were conducted with hatcheryreared fish in freshwater.

Many of the studies that failed to document mortality may not have used dietary concentrations that were sufficiently high or experimental durations that were sufficiently long to induce mortality. The lowest documented LOAEL (1.9 $\mathrm{gg} \mathrm{g}^{-1}$ wet wt) induced mortality of male killifish (Fundulus heteroclitus), but aggressive behavior exhibited by the male fish may have also contributed to early mortality (Supplemental Reference S11). The next closest LOAEL $\left(4.1 \mu \mathrm{gg}^{-1}\right.$ wet wt) for juvenile beluga sturgeon (Supplemental Reference S4) is nearly twofold higher, and the threshold effect level $\left(2.8 \mu \mathrm{gg}^{-1}\right.$ wet wt) may provide a better estimation of the threshold value. It is difficult to assess the applicability of thresholds derived for lethal effects here to $\mathrm{MeHg}$ burdens in prey that might induce lethal effects in the field. To our knowledge, only anecdotal observations of mortality exist for highly contaminated ecosystems (e.g., Minamata Bay, Japan, during the 1950s [5] and the English-Wabigoon River, Ontario, Canada, during the 1970s [30]). Given the high concentrations of $\mathrm{Hg}$ measured in a variety of organisms at that time, which substantially exceeded $1.0 \mu \mathrm{gg}^{-1}$ wet weight at these grossly polluted sites [30], mortality due to $\mathrm{MeHg}$ exposure is certainly a plausible outcome. Takeuchi [5], for example, reported that "a number of fish died" and that fish in Minamata Bay "frequently could easily be captured by hand," indicating severely diminished locomotor activity and escape behavior. Fish from the bay were commonly emaciated and had lesions in the brain [5].

\section{High ecological risk: Growth and reproduction}

Differences in sensitivity between reproductive and growth endpoints may reflect a disproportionate availability of studies that report growth endpoints (nine) compared to the five that reported reproductive endpoints. Nonetheless, adverse effects on reproduction occurred at dietary $\mathrm{MeHg}$ concentrations below $0.5 \mu \mathrm{g} \mathrm{g}^{-1}$ wet weight, whereas adverse effects related to growth occurred at concentrations exceeding $2.5 \mu \mathrm{gg}^{-1}$ wet weight; and many studies yielded unbounded NOAEL (Fig. 2). 
Table 3. Summary of dietary threshold concentrations of $\mathrm{MeHg}$ for fish $\left(\mu \mathrm{gg}^{-1}\right.$ wet wt)

\begin{tabular}{|c|c|c|c|c|c|}
\hline & Highest NOAEL & Lowest LOAEL & TEL & Proposed threshold & No. of species \\
\hline Growth & 1.44 & 1.97 & 2.15 & 1.44 & 10 \\
\hline Reproductive & 0.04 & 0.05 & NA & 0.04 & 4 \\
\hline Biochemical & 0.06 & 0.14 & 0.18 & 0.06 & 7 \\
\hline
\end{tabular}

Highest NOAEL $=$ the highest-ranked no observed adverse effect level (NOAEL) below the lowest observed adverse effect level (LOAEL); lowest LOAEL = the lowest-ranked LOAEL; TEL = calculated threshold effect level (see Methods section); proposed threshold = the proposed threshold level; No. test species $=$ the number of species used to derive each quantity.

The most common manifestation of reduced growth was a reduction in fish size or weight gain (Supplemental References S4, S5 [2\% ration group only], S7, S14-S17), although at least one study reported increased weights in fish exposed to $\mathrm{MeHg}$ (Supplemental Reference S8). The severity of effects appeared to increase with experimental duration and/or dietary $\mathrm{MeHg}$ concentration. For example, adult walleye fed $7.9 \pm 0.7 \mu \mathrm{g} \mathrm{g}^{-1}$ wet weight $\mathrm{MeHg}$ for more than $296 \mathrm{~d}$ were severely emaciated (Supplemental Reference S13) compared to juvenile walleye that exhibited only reduced weight gain when fed a lower dietary $\mathrm{MeHg}$ concentration $\left(0.987 \mu \mathrm{gg}^{-1}\right.$ wet wt) for a shorter duration (180d) (Supplemental Reference S14). Likewise, reductions in growth rate were observed at earlier stages in green sturgeon fed $24.3 \mu \mathrm{g} \mathrm{g}^{-1}$ wet weight (four weeks) compared to those fed $9.7 \mu \mathrm{gg}^{-1}$ wet weight (eight weeks) or to controls (Supplemental Reference S15).

Reductions in growth may result from the diversion of resources from somatic growth to physiological maintenance (Supplemental Reference S17) or to reductions in nutrient uptake as a result of increased gut motility (Supplemental References S15,S17). In vitro experiments have demonstrated $\mathrm{MeHg}$-induced inhibition of L-leucine absorption in the intestine of toadfish (Opsanus tau) [31], and observations of increased fecal matter production in some experiments support this hypothesis (Supplemental References S15,S17). Reduced growth also may be linked to $\mathrm{MeHg}$-induced visual impairment. In several studies that observed emaciation or reduced weight gain, behavioral aberrations manifested as abnormal responses to light stimulus (Supplemental Reference S13), impaired spatial awareness, and lethargy were noted (Supplemental References S5,S13,S16). Methylmercury is known to impair both color vision and contrast sensitivity in rainbow trout (Oncorhynchus mykiss) [32] and trahira (Hoplias malabaricus) [33]. More recently, deposition of $\mathrm{MeHg}$ granules in the central portion of the retina of zebrafish (Danio rerio) fed the same diets used in the zebrafish studies reviewed here (Table 2) has been demonstrated [34]. Both photopic and scotopic sensitivity are important visual components for successful foraging and prey capture [35]; therefore, impairment of vision may lead to reduced prey or food-capture ability and subsequently to reduced growth. The reduction in food consumption observed in some experiments (Supplemental References S4,S6) may be one manifestation of visual impairment, but potential confounding effects of ration size and/or digestibility cannot be separated at present.

The ranking approach and calculated threshold effect level yielded threshold values for reductions in growth or weight gain of 1.4 and $2.2 \mu \mathrm{g} \mathrm{g}^{-1}$ wet weight, respectively (Table 3 ). Like mortality, adverse effects on growth or weight gain are difficult to assess in fish populations in the field due to the multitude of confounding factors that can influence the growth of fishes.
However, Lockhart et al. [6] noted that northern pike (Esox lucius) ranging in weight from 4 to $18 \mathrm{~kg}$ collected in 1971 from Clay Lake, Ontario, Canada, were severely emaciated. While these investigators did not collect smaller prey fish, Vermeer et al. [36] measured a mean $\mathrm{Hg}$ concentration in 10 whole yellow perch (Perca flavescens) collected from stomachs of common mergansers (Mergus merganser) on Clay Lake in the same year of $2.7 \mu \mathrm{gg}^{-1}$ (range $1.3-3.6 \mu \mathrm{gg}^{-1}$ wet wt). Although these concentrations exceed the derived threshold for lethal effects, they also exceed our threshold value where we expect to observe adverse effects on growth (Table 3). In contrast, Hall et al. [13] failed to observe any adverse effect on growth or survival in redside dace (Clinostomus elongatus) grazing on zooplankton with $\mathrm{MeHg}$ concentrations ranging from 0.02 to $0.08 \mu \mathrm{g} \mathrm{g}^{-1}$ wet weight, concentrations well below the threshold for growth. Although limited, these results are consistent with our derived value for growth-related endpoints.

Only five studies attempted to assess reproductive endpoints. Effects were observed at dietary $\mathrm{MeHg}$ concentrations below $0.5 \mu \mathrm{gg}^{-1}$ wet weight (Table 2 and Fig. 2). For reproductive endpoints, only four species were available, so calculation of the threshold effect level was not possible (Table 3). Therefore, we use the highest-ranked unbounded NOAEL for walleye of $0.04 \mu \mathrm{g} \mathrm{g}^{-1}$ wet weight as the protective threshold. The reproductive axis in fish is thought to be sensitive to $\mathrm{MeHg}$ [37], with documented effects including reductions in fecundity (Supplemental Reference S11), spawning success (Supplemental References S8-S10), circulating sex steroids (Supplemental References S10,S18), time spent spawning (Supplemental Reference S10), and gonadosomatic index (GSI) (Supplemental References S8,S9,S14). Drevnick et al. (Supplemental Reference S18) suggest that MeHg induces apoptosis in steroidogenic cells in the gonads, leading to some of the effects described above. For coregonids, salmonids, and percids, GSI is generally an accepted measure of reproductive capacity because they spawn typically once per year; however, for cyprinid species (e.g., fathead minnows), GSI may vary considerably among spawning cycles [38]. However, the response of GSI observed in the fathead minnow studies (Supplemental References S8,S9) is consistent with other reproductive endpoints in the same studies (Table 2) and with the trend of GSI measured in juvenile walleye (Supplemental Reference S14).

Comparable field evidence for reproductive endpoints is limited, but reductions in testosterone have been observed in male largemouth bass (Micropterus salmoides) in the Manasquan Reservoir, New Jersey, USA [12]. Concentrations of $\mathrm{Hg}$ in prey fish from the same location were above the suggested threshold for adverse effects (yellow perch $<20 \mathrm{~cm}, 0.13 \mu \mathrm{g} \mathrm{g}^{-1}$; bluegill [Lepomis macrochirus] $<17 \mathrm{~cm}, \quad 0.24 \mu \mathrm{gg}^{-1} ;$ alewife [Alosa pseudoharengus] $<16 \mathrm{~cm}, 0.41 \mu \mathrm{g} \mathrm{g}^{-1}$ —all wet wt [39]). Reductions in plasma 
testosterone and GSI also have been observed in immature male white sturgeon (Acipenser transmontanus) in the Columbia River when muscle and gonad $\mathrm{Hg}$ content exceeded 0.19 and $0.07 \mu \mathrm{gg}^{-1}$ wet weight, respectively, suggesting that $\mathrm{MeHg}$ levels in prey need not be exceptionally high to potentially impact reproduction [40].

Transgenerational effects of maternally derived $\mathrm{MeHg}$ are relatively understudied (Supplemental Reference S8,S11,S12). Early life stages of fish are demonstrably sensitive to $\mathrm{MeHg}$ in aqueous exposures [41,42], but the dominant source of $\mathrm{MeHg}$ exposure at these developmental stages is directly linked to the diet of the female during oogenesis [43]. At present, only two studies have documented evidence of transgenerational effects of $\mathrm{MeHg}$. Reduced fecundity and skewed sex ratios in offspring were observed in subsequent generations of killifish (Supplemental Reference S11), while negative impacts on predator evasion and foraging success of Atlantic croaker (Micropogonias undulatus) larvae were suggested via computer modeling (Supplemental Reference S12). Hammerschmidt et al. (Supplemental Reference S8) did not observe any adverse effects in fathead minnow larvae hatched from trophically contaminated adults, but the duration of follow-up was very short (7d) and may not have been sufficient to detect adverse effects in subsequent generation spawning success or larval behavior. Additional work is required to further elucidate the scope of adverse effects in this context given that such effects may be long-lasting, if not permanent [44].

Moderate risk: Histological, biochemical, and neurobehavioral effects

Histological, biochemical, and genomic effects appear to occur at dietary $\mathrm{MeHg}$ concentrations comparable to those at which reproductive effects are observed $\left(<0.5 \mu \mathrm{g} \mathrm{g}^{-1}\right)$ but vary with the sensitivity of specific test protocols. Most of the endpoints are associated with evidence of oxidative stress, ranging from structural damage in tissues or organs to differential gene transcription (Table 2). Histological examinations revealed lesions in brain tissue and central nervous tissue (Supplemental References S6,S16,S19), intestinal lining (Supplemental Reference S4,S7,S20), and disrupted muscle fibers (Supplemental Reference S21). The presence of increased numbers of melano-macrophage centers in a variety of organs including liver and head kidney (Supplemental Reference S3) and increased glutathione system enzyme activity in brain and liver tissue (Supplemental Reference S19) are also consistent with increased oxidative stress and the presence of reactive oxygen species. Induction of genes related to oxidative stress defense systems has been observed in some species (Supplemental References S22,S23) but not in others (Supplemental Reference S24), although this may be related to differences in experimental dosing and exposure regimes. Inductions of genes associated with endocrine disruption have been observed in fathead minnows (Supplemental Reference S24) and are consistent with the suppression of sex steroids and reproduction in the same species (Supplemental Reference S9). Discoloration of the skin is commonly reported in rainbow trout (Supplemental References S5,S7,S16), and disruption of metabolic processes in the mitochondria (Supplemental Reference S25) and reduced $\mathrm{O}_{2}$ consumption (Supplemental Reference S17) have also been observed.

The ranked NOAEL and calculated threshold effect level for histological, biochemical, and genomic effects are 0.06 and $0.18 \mu \mathrm{gg}^{-1}$ wet weight, respectively (Table 3 ). The discrepancy between these values can be partly explained by the large differences in sensitivity among endpoint measures and a much larger range of $\mathrm{MeHg}$ concentrations in the experimental diets used in the studies. Although field studies documenting similar sublethal effects have not reported $\mathrm{MeHg}$ in coexisting prey, the $\mathrm{Hg}$ concentrations in some of the test fish are quite low and, by extension, the diet also is likely characterized by low $\mathrm{MeHg}$ concentrations. For example, Drevnick et al. [7] noted increased lipofuscin in the livers of northern pike from lakes on Isle Royale, in Lake Superior, Michigan, USA. While MeHg concentrations in forage fish are not available for all lakes from the same time period, levels of total $\mathrm{Hg}$ in yellow perch from two of the lakes where Drevnick et al. [7] observed high $\mathrm{Hg}$ in northern pike (Sargeant and Richie Lakes) ranged from 0.019 to $0.070 \mu \mathrm{g} \mathrm{g}^{-1}$ wet weight (3-18 cm in total length [45]). Given that $\mathrm{Hg}$ concentrations appear to have declined since the mid- to late 1990s in these lakes [46], it seems likely that concentrations in yellow perch and other forage fish were even lower in 2004 to 2006, when Drevnick et al. [7] sampled northern pike at Isle Royale. Schwindt et al. [9] documented an increase in melanomacrophage centers in spleen and kidney of brook trout (Salvelinus fontinalis) from 14 lakes in the western United States. Whole-body concentrations of $\mathrm{Hg}$ in brook trout ranged from approximately 0.03 to $0.29 \mu \mathrm{g} \mathrm{g}^{-1}$ wet weight. Similarly, upregulation of genes related to oxidative stress and endocrine responses was associated with $\mathrm{Hg}$ concentrations of 0.016 to $0.054 \mu \mathrm{gg}^{-1}$ wet weight in cutthroat trout (Oncorhynchus clarkii) [11]. Given the relatively low $\mathrm{Hg}$ burdens in the fish from these studies, we surmise that the $\mathrm{MeHg}$ concentrations in the diets of these fish are also low and therefore consistent with the low threshold value.

The ranked threshold and calculated threshold effect level for behavioral endpoints are relatively similar $(0.50$ and $0.61 \mu \mathrm{g} \mathrm{g}^{-1}$ wet wt, respectively). Although nearly an order of magnitude higher than more sensitive endpoints related to histology, biochemistry, or genotoxicity, this most likely reflects the difference between the presence of subclinical effects of $\mathrm{MeHg}$ exposure and the accumulated subclinical damage required to elicit behavioral changes at the organism level. Behavioral abnormalities linked to $\mathrm{MeHg}$ exposure are thought to be due to interference with neurotransmitter production, receptors, or cell signaling pathways [47]. However, the behavioral endpoints in many of the fish species tested to date are characterized by observational data on organism activity or stimulus-response experiments. The behavioral abnormalities most frequently reported are related to increased lethargy and altered resting position (Supplemental References $\mathrm{S} 5, \mathrm{~S} 7, \mathrm{~S} 16, \mathrm{~S} 19)$. Obvious impairment of locomotion is commonly observed in mammals and birds exposed to $\mathrm{MeHg}$ [3] but has been observed in fish in only one study to date (Supplemental Reference S16). Abnormal responses to light (Supplemental Reference S13) and altered shoaling and dispersal behavior (Supplemental Reference S6) represent affected stimulus-response behavior and may increase susceptibility to predation (Supplemental Reference S6), but the level of impairment of behavior that constitutes a significant risk is unclear and may require evaluation through modeling (Supplemental Reference S12) or direct experimentation (Supplemental Reference S6).

In fish-eating mammals and birds, novel approaches are currently being used to assess subclinical neurological damage linked to $\mathrm{MeHg}$ exposure $[48,49]$. For example, reductions in $\mathrm{N}$ methyl-D-aspartate receptors may exert excitotoxic effects due to a buildup of glutamate in extracellular spaces [48,50], and disruption of various elements of the cholinergic system in 
mammals [51] have been observed at lower doses of $\mathrm{MeHg}$ than those that cause overt symptoms of neurological damage or abnormal behavior. Similar work in fish is uncommon, but Adams et al. [52] recently reported on an association of reduced $\mathrm{N}$-methyl-D-aspartate receptor levels and $\mathrm{MeHg}$ in spotted sea trout (Cynoscion nebulosus) in south Florida, suggesting that these novel tools may offer a more sensitive means to diagnose behavioral effects of $\mathrm{MeHg}$ exposure that may occur at lower concentrations.

Determining the $\mathrm{MeHg}$ thresholds for sublethal effects is a first and necessary step of risk assessment. However, sublethal effects are difficult to link to population impacts because they often do not directly translate into population-relevant endpoints such as survival and reproduction [53]. Recent efforts that use an adverse outcome approach that embeds sublethal effects into a computational framework and/or provides a means to interpret sublethal effects at the population level will determine whether such adverse effects measured at the behavioral or cellular level impact population health [54]. As the adverse outcome pathways become better characterized in future studies, the sublethal endpoints related to dietary $\mathrm{MeHg}$ exposure reported here will become increasingly relevant to assess fish population health.

Factors contributing to variation in dietary $\mathrm{MeHg}$ toxicity: Species and life stage

Adverse effects due to dietary $\mathrm{MeHg}$ exposure and the levels at which they occur appear to vary considerably among species (Supplemental Reference S15). The apparent variation in sensitivity may be partly attributable to use of both adult and juvenile data within species, differences in experimental protocols, endpoint measurement techniques and sensitivity, and species-specific digestive physiology. Assimilation efficiency of $\mathrm{MeHg}$ from the diet in most fish is generally thought to exceed $80 \%$, particularly for fish ingesting naturally contaminated prey [55]. In laboratory experiments, assimilation efficiency can range considerably (50-94\% [56] (Supplemental References S5,S17,S20) and may be affected not only by the species digestive physiology but also perhaps by the experimental diet formulation and dosing regime.

In carnivorous and omnivorous fish, digestion takes place in the acidic environment of the stomach $(\mathrm{pH} \sim 2)$. However, some cyprinid species (e.g., Sacramento blackfish [Orthodon microlepidotus], fathead minnow) do not have a true stomach and have a digestive system that is neutral to alkaline throughout [57]. This may lead to intraspecific differences in $\mathrm{MeHg}$ availability from experimental diets. Using in vitro digestions, Leaner and Mason [58] observed significant differences in $\mathrm{MeHg}$ availability between Atlantic sturgeon and channel catfish (Ictalurus punctatus) and suggested that different amounts of amino acids in digestive juices may influence assimilation of $\mathrm{MeHg}$. Goto and Wallace [59] have recently demonstrated that assimilation efficiency can vary substantially within a single species (e.g., 50-90\%) depending on the composition of the diet. Uptake of $\mathrm{MeHg}$ across the intestinal wall also appears to be temperature-dependent [60] and, all other factors being equal, implies a greater uptake and assimilation of $\mathrm{MeHg}$ under warm-water experimental conditions compared to those typically used for cold-water species such as rainbow trout.

\section{Differences in experimental protocols: Form of administered $\mathrm{MeHg}$}

It has long been accepted that the chemical form of $\mathrm{MeHg}$ in fish tissues is largely $\mathrm{MeHg}$ bound to sulfhydryl groups in proteins [61]. Recent work has suggested that the dominant form of $\mathrm{MeHg}$ in muscle tissue in marine and freshwater fish is methylmercury cysteinate (MeHgCys), where $\mathrm{MeHg}$ is bound to the thiol group on cysteine [62]. However, nearly all the dietary exposure studies examined here utilized diets amended with $\mathrm{MeHgCl}$ (with the exception of Scherer et al. [Supplemental Reference S13]), mainly on the premise that this mimics the form of $\mathrm{MeHg}$ present in the stomach after acidic digestion [58]. In vitro experiments suggest that $\mathrm{MeHg}$ remains bound to cysteine after digestion with gastric fluids [63] and that the highly lipophilic nature of $\mathrm{MeHgCl}$ compared to $\mathrm{MeHgCys}$ may permit rapid translocation across the intestinal membrane [64], whereas MeHgCys appears to require the assistance of $\mathrm{L}_{-}$ specific neutral amino acid transporters [60]. While this might imply differential uptake and/or toxicity of $\mathrm{MeHgCl}$ compared to $\mathrm{MeHgCys}$, evidence to date suggests that $\mathrm{MeHgCl}$ from experimentally spiked diets likely complexes with amino acids present in digestive fluids and crosses the membrane using amino acid transporters $[58,60]$. Dietary exposures of naturally contaminated food versus spiked food reveal contrasting results depending on the organism in question; Berntssen et al. [65] observed higher excretion and lower accumulation of $\mathrm{MeHg}$ from naturally contaminated fish compared to $\mathrm{MeHgCl}$ amended diets in Wistar rats, while the opposite pattern was observed for largemouth bass (Micropterus salmoides) fed naturally contaminated crayfish and $\mathrm{MeHgCl}$-amended commercial fish food [66]. At present, insufficient evidence exists to conclude that one form of dietary $\mathrm{MeHg}$ is more or less toxic than the other, but these differences may merit consideration in planning and interpreting future studies.

\section{Dietary Se and implications for $\mathrm{MeHg}$ toxicity}

The ability of Se to ameliorate adverse effects of $\mathrm{MeHg}$ has been recognized for decades and undergone extensive evaluation in animal models [24,25]. Ralston and Raymond [67] present a conceptual model of $\mathrm{MeHg}$ and Se interaction. In this model, $\mathrm{MeHg}$ and $\mathrm{Hg}$ act as irreversible selenoenzyme inhibitors [68]. On entry to the cell, MeHgCys is thought to form $\mathrm{MeHgSeCys}$ ("pseudomethionine" [67]), which directly inhibits selenoenzymes and traps Se, making it unavailable for further cycles of selenocysteine synthesis [67]. Consequently, some, if not all, of the symptoms of $\mathrm{MeHg}$ toxicity are a manifestation of a gradual depletion of Se from internal Se pools coupled with an increasing impairment of Se-dependent enzyme activities, particularly as the $\mathrm{Hg}$ :Se molar ratio exceeds 1.0 [67]. This model has considerable support in the mammalian literature [25], but comparable studies on fish are scarce. Turner and Swick [69] provide corroborating evidence that Se accumulated through the diet can reduce $\mathrm{MeHg}$ accumulation in northern pike, and similar effects are inferred from patterns of $\mathrm{MeHg}$ and Se accumulation in yellow perch from lakes near Sudbury, Ontario, Canada [70]. In contrast, Dang and Wang [71] suggest that Se (independent of chemical species) does not affect $\mathrm{MeHg}$ accumulation from the diet in a marine fish (Terapon jarbua). Despite these contrasting results, evidence for amelioration of adverse effects in fish remains limited. To our knowledge, only one study has examined the effect of coadministered dietary $\mathrm{Se}$ and $\mathrm{MeHg}$ to fish in a controlled setting. Deng et al. [72] demonstrated that dietary Se reduced $\mathrm{MeHg}$ accumulation and inhibited histological damage to tissues in Sacramento splittail (Pogonichthys macrolepidotus) larvae when fed both $\mathrm{MeHg}$ and Se in moderate concentrations. Future experimental work would benefit from an assessment of the Se content in experimental diets. 


\section{CONCLUSIONS AND RECOMMENDATIONS}

The thresholds presented here, to our knowledge, are the first to define dietary $\mathrm{MeHg}$ thresholds for fish. Although these should be considered preliminary due to the limited information available, this overview provides a baseline for future experimental and mensural studies. It is important to note that the present study and the thresholds derived herein are based entirely upon laboratory experiments and were generally conducted under favorable conditions. Such conditions are unlikely to be present in the field, where additional environmental stressors related to foraging, predation, temperature fluctuation, and other potentially toxic contaminants are present [73]. Thus, our thresholds defined here, albeit intentionally conservative, may still underestimate the magnitude of effects experienced by wild fish.

Although recent advances in understanding the effects of dietary $\mathrm{MeHg}$ exposure on fish-eating birds and mammals have been impressive (reviewed in Scheuhammer et al. [3]), the lack of high-quality toxicological studies on the adverse effects of dietary $\mathrm{MeHg}$ exposure to fish and the assumptions used in the present study somewhat limit the level of confidence that can be ascribed to the thresholds reported here. However, we feel that these thresholds are both useful and relevant because our thresholds are in general agreement with the limited available evidence from field studies and are in the same relative magnitude for other vertebrate species, which have been studied more extensively. For example, Shore et al. [74] suggest a lethal threshold of $\sim 6$ and $0.9 \mu \mathrm{g} \mathrm{g}^{-1}$ wet weight for 11 species of birds and nine species of mammals and a reproductive threshold of $0.25 \mathrm{\mu g} \mathrm{g}^{-1}$ wet weight for seven species of nonmarine birds. It is worth noting that, based on additional research, the reproductive threshold described by Shore et al. [74] is nearly an order of magnitude lower than previously thought [75], which suggests that continued study will likely result in further refinement and improvement in our thresholds as well.

From this review and others [16,74], reproductive effects appear to be the most sensitive population-level endpoints in vertebrates, and reproductive studies on additional fish species would be worthwhile. Furthermore, because early life stages appear to be more sensitive to $\mathrm{MeHg}$ compared to the adult stage [17], experiments to assess the magnitude and longevity of adverse effects on developmental or behavioral impacts in embryo and larval stages under dietary exposures remain a worthwhile avenue of research. Efforts to link subclinical biomarkers of $\mathrm{MeHg}$ toxicity to individual-level effects [76] will be essential for establishing quantitative relationships and aiding the interpretation of the rapidly expanding literature base documenting various subclinical effects of $\mathrm{MeHg}$ exposure in fish.

Consideration of the diet composition and formulation may also be important for comparing among species and relevant endpoints, and field studies should take into consideration the $\mathrm{MeHg}$ content of likely prey items, which may necessitate the use of stable isotopes or direct sampling of prey, but also the presence of other contaminants or stressors. We echo the sentiment of Beckvar et al. [17], that future laboratory work use rigorously designed experiments, such as those used by Hammerschmidt et al. (Supplemental Reference S8) and Berntssen et al. (Supplemental Reference S19), that provide multiple and realistic dosing levels, a variety of suitable endpoints, and dietary exposure to $\mathrm{MeHg}$. These careful approaches, coupled with the development of more refined and sensitive methods that can be used to measure $\mathrm{MeHg}$ effects in the field, will contribute to a better understanding of the risks of $\mathrm{MeHg}$ to wild fish.

Acknowledgement-The present study is the result of a two-day workshop held at the University of Michigan in July 2010. The authors thank K. Williams and D. Evers (Biodiversity Research Institute) for logistical assistance with the workshop and J. Rutkiewicz (University of Michigan) for a meticulous recording of the workshop discussions. Funding for the workshop was provided by Environment Canada. Comments from two anonymous reviewers are appreciated.

\section{REFERENCES}

1. Wiener JG, Krabbenhoft DP, Heinz GH, Scheuhammer AM. 2003. Ecotoxicology of mercury. In Hoffman DJ, Rattner BA, Burton GA Jr, Cairns J Jr, eds, Handbook of Ecotoxicology, 2nd ed. Lewis, Boca Raton, FL, USA, pp 409-463.

2. Harris RC, Rudd JW, Amyot M, Babiarz CL, Beaty KG, Blanchfield PJ, Bodaly RA, Branfireun BA, Gilmour CC, Graydon JA, Heyes A, Hintelmann H, Hurley JP, Kelly CA, Krabbenhoft DP, Lindberg SE, Mason RP, Paterson MJ, Podemski CL, Robinson A, Sandilands KA, Southworth GR, St Louis VL, Tate MT. 2007. Whole ecosystem study shows rapid fish mercury response to changes in mercury deposition. Proc Natl Acad Sci USA 104:16586-16591.

3. Scheuhammer AM, Meyer MW, Sandheinrich MB, Murray MW. 2007. Effects of environmental methylmercury on the health of wild birds mammals, and fish. Ambio 36:12-18.

4. U.S. Environmental Protection Agency. 1997. Mercury Study Report to Congress. Vol 7. Characterization of Human Health and Wildlife Risks from Mercury Exposure in the Unites States. Washington, DC.

5. Takeuchi T. 1968. Pathology of Minamata disease. In Study Group of Minamata Disease, ed, Minamata Disease. Medical School of Kumamoto University, Kumamoto, Japan, pp 141-228.

6. Lockhart WL, Uthe JF, Kenney AR, Mehrle M. 1972. Methylmercury in northern pike (Esox lucius): Distribution, elimination, and some biochemical characteristics of contaminated fish. J Fish Res Board Can 29:1519-1523.

7. Drevnick PE, Roberts AP, Otter RR, Hammerschmidt CR, Klaper R, Oris JT. 2008. Mercury toxicity in livers of northern pike (Esox lucius) from Isle Royal, USA. Comp Biochem Physiol C Toxicol Pharmacol 147:331338.

8. Larose C, Canuel R, Lucotte M, Di Giulio RT. 2008. Toxicological effects of methylmercury on walleye (Sander vitreus) and perch (Perca flavescens) from lakes of the boreal forest. Comp Biochem Physiol C Toxicol Pharmacol 147:139-149.

9. Schwindt A, Fourne JW, Landers DH, Schreck CB, Kent ML. 2008. Mercury concentrations in salmonids from western US national parks and relationships with age and macrophage aggregates. Environ Sci Technol 42:1365-1370.

10. Mieiro CL, Ahmad I, Pereira ME, Duarte AC, Pacheco M. 2010. Antioxidant system breakdown in brain of feral golden grey mullet (Liza aurata) as an effect of mercury exposure. Ecotoxicology 19:10341045.

11. Moran PW, Aluru N, Black RW, Vijayan MM. 2007. Tissue contaminants and associated transcriptional response in trout liver from high elevation lakes of Washington. Environ Sci Technol 41:65916597.

12. Friedmann AS, Costain EK, MacLatchy DL, Stansley W, Washuta EJ. 2002. Effect of mercury on general and reproductive health of largemouth bass (Micropterus salmoides) from three lakes in New Jersey. Ecotoxicol Environ Saf 52:117-122.

13. Hall BD, Bodaly RA, Fudge RJP, Rudd JWM, Rosenberg DM. 1997. Food as the dominant pathway of methylmercury uptake by fish. Water Air Soil Pollut 100:13-24.

14. McKim JM, Olson GF, Holcombe GW, Hunt FP. 1976. Long term effects of methylmercuric chloride on three generations of brook trout (Salvelinus fontinalis): Toxicity, accumulation, distribution, and elimination. J Fish Res Board Can 33:2727-2739.

15. Wester PW, Canton HH. 1992. Histopathological effects in Poecilia reticulata (guppy) exposed to methyl mercury chloride. Toxicol Pathol 20:81-92.

16. Sandheinrich MB, Wiener JG. 2011. Methylmercury in freshwater fish-Recent advances in assessing toxicity of environmentally relevant exposures. In Beyer WN, Meador JP, eds, Environmental Contaminants 
in Biota: Interpreting Tissue Concentrations, 2nd ed. CRC/Taylor and Francis, Boca Raton, FL, USA, pp 169-190.

17. Beckvar N, Dillon TM, Read LB. 2005. Approaches for linking wholebody fish tissue residues of mercury or DDT to biological effects thresholds. Environ Toxicol Chem 24:2094-2105.

18. Dillon TM, Beckvar N, Kern J. 2010. Residue-based mercury doseresponse in fish: An analysis using lethality equivalent test endpoints. Environ Toxicol Chem 29:2559-2565.

19. Buekers J, Steen Redeker E, Smolders E. 2009. Lead toxicity to wildlife: Derivation of a critical blood concentration for wildlife monitoring based on literature data. Sci Total Environ 407:3431-3438.

20. Clarkson TW, Magos L, Myers GJ. 2003. The toxicology of mercuryCurrent exposures and clinical manifestations. N Engl J Med 349:17311737.

21. Barrows FT, Hardy RW. 2001. Nutrition and feeding. In Wedenmeyer G, ed, Fish Hatchery Management, 2nd ed. American Fisheries Society, Bethesda, MD, USA, pp 483-558.

22. Rodgers DW, Qadri SU. 1982. Growth and mercury accumulation in yearling yellow perch, Perca flavescens, in the Ottawa River, Ontario. Environ Biol Fish 7:377-383.

23. Eisler R. 1985. Selenium hazards to fish, wildlife and invertebrates: A synoptic review. Contaminant Hazard Reviews 1-35. Patuxent Wildlife Research Center, Laurel, MD, USA.

24. Ganther HE, Goudie C, Sunde ML, Kopecky MJ, Wagner P, Oh S-H Hoekstra WG. 1972. Selenium: Relation to decreased toxicity of methylmercury added to diets containing tuna. Science 175:1122-1124.

25. Ganther HE, Sunde ML. 2007. Factors modifying methylmercury toxicity and metabolism. Biol Trace Elem Res 119:221-233.

26. Subcommittee on Fish Nutrition, National Research Council. 1993. Nutritional Requirements of Fish. National Academy Press, Washington, DC

27. Choi MH, Cech JJ. 1998. Unexpectedly high mercury level in pelleted commercial fish feed. Environ Toxicol Chem 17:1979-1981.

28. Grist EPM, Leung KMY, Wheeler JR, Crane M. 2002. Better bootstrap estimation of hazardous concentration thresholds for aquatic assemblages. Environ Toxicol Chem 21:1515-1524.

29. Canadian Council of Ministers of the Environment. 1995. Protocol for derivation of the Canadian sediment quality guidelines for the protection of aquatic life. CCME-EP98E. Ottawa, ON.

30. Fimreite N, Reynolds LM. 1973. Mercury contamination of fish in northwestern Ontario. J Wildl Manage 37:62-68.

31. Farmanfarmaian AV, Socci R. 1984. Inhibition of essential amino acid absorption in marine fishes by mercury. Mar Environ Res 14:185-199.

32. Hawryshyn CW, Mackay WC, Nilsson TH. 1982. Methylmercury induces visual deficits in rainbow trout. Can J Zool 60:3127-3133.

33. Tanan CL, Ventura DF, de Souza JM, Grötzner SR, Mela M, Gouveia A 2006. Effects of mercury intoxication on the response of horizontal cells of the retina of trahira fish (Hoplias malabaricus). Braz J Med Biol Res 39:987-995.

34. Mela M, Cambier S, Mesmer-Dudons N, Legay A, Grötzner SR, OliviaRibeiro CAD, Ventura DF, Massabuau J-C. 2010. Methylmercury localization in Danio rerio retina after trophic and subchronic exposure: A basis for neurotoxicology. Neurotoxicology 31:448-453.

35. Douglas RH, Hawryshyn CW. 1990. Behavioural studies of fish vision: An analysis of visual capabilities. In Douglas RH, Djamqoz MBA, eds, The Visual System of Fish. Chapman and Hall, London, UK, pp 373-417.

36. Vermeer K, Armstrong FAJ, Hatch DRM. 1973. Mercury in aquatic birds at Clay Lake, western Ontario. J Wildl Manage 37:58-61.

37. Crump KL, Trudeau VL. 2009. Mercury-induced reproductive impairment in fish. Environ Toxicol Chem 28:895-907.

38. Fox MG, Crivelli AJ. 1998. Body size and reproductive allocation in a multiple spawning centrarchid. Can J Fish Aquat Sci 55:737-748.

39. Horwitz RJ, Velinsky D, Overbeck P, Kiry P. 1999. Phase II assessment of total mercury concentrations in fishes from rivers, lakes and reservoirs of New Jersey. Report 99-7R. Patrick Center for Environmental Research, Academy of Natural Sciences of Philadelphia, Philadelphia, PA, USA.

40. Webb MAH, Feist GW, Fitzpatrick MS, Foster EP, Schreck CB, Plumlee M, Wong C, Gundersen DT. 2006. Mercury concentrations in the gonad, liver and muscle of white sturgeon (Acipenser transmontanus) in the lower Columbia River. Arch Environ Contam Toxicol 50:443-451.

41. Latif MA, Bodaly RA, Johnston TA, Fudge RJP. 2001. Effects of environmental and maternally derived methylmercury on the embryonic and larval stages of walleye (Stizostedion vitreum). Environ Pollut 111:139-148

42. Devlin EW. 2006. Acute toxicity, uptake and histopathology of aqueous methylmercury to fathead minnow embryos. Ecotoxicology 15:97-110.
43. Hammerschmidt CR, Sandheinrich MB. 2005. Maternal diet during oogenesis is the major source of methylmercury in fish embryos. Environ Sci Technol 39:3580-3584.

44. Fjeld E, Haugen TO, Vøllestad LA. 1998. Permanent reductions in the foraging efficiency and competitive ability of grayling (Thymallus thymallus) exposed to methylmercury during embryogenesis. Sci Total Environ 213:247-254.

45. Gorski PG, Cleckner LB, Hurley JP, Sierszen ME, Armstrong DE. 2003. Factors affecting enhanced mercury bio-accumulation in inland lakes of Isle Royale National Park, USA. Sci Total Environ 304:327-348.

46. Drevnick PE, Canfield DE, Gorski PR, Shinneman ALC, Engstrom DR, Muir DCG, Smith GR, Garrison PJ, Cleckner LB, Hurley JP, Noble RB, Otter RR, Oris JT. 2007. Deposition of sulphur controls mercury accumulation in Isle Royale fish. Environ Sci Technol 41:7266-7272.

47. Stamler CJ, Basu N, Chan HM. 2005. Biochemical markers of neurotoxicity in wildlife and human populations: Considerations for method development. J Toxicol Environ Health A 68:1413-1429.

48. Basu N, Scheuhammer AM, Rouvinen-Watt K, Grochowina N, Evan RD, O'Brien M, Chan HM. 2007. Decreased $N$-methyl-D-aspartic acid (NMDA) receptor levels are associated with mercury exposure in wild and captive mink. Neurotoxicology 28:587-593.

49. Scheuhammer AM, Basu N, Burgess NM, Elliot JE, Campbell GD, Wayland M. 2008. Relationships among mercury, selenium, and neurochemical parameters in common loons (Gavia immer) and bald eagles (Haliaeetus leucocephalus). Ecotoxicology 17:93-101.

50. Basu N, Scheuhammer AM, Sonne C, Letcher RJ, Born EW, Dietz R. 2009. Is dietary methylmercury of neurotoxicological concern to polar bears (Ursus maritimus)? Environ Toxicol Chem 28:133-140.

51. Basu N, Scheuhammer AM, Rouvinen-Watt K, Grochowina N, Klenavic K, Evans D. 2006. Methylmercury impairs components of the cholinergic system in captive mink (Mustela vison). Toxicol Sci 91 : 202-209.

52. Adams DH, Sonne C, Basu N, Nam D-H, Leifsson PS, Jensen AL. 2010. Mercury contamination in spotted seatrout Cynoscion nebulosus: An assessment of liver, kidney, blood and nervous system health. Sci Total Environ 408:5808-5816.

53. Kramer VJ, Etterson MA, Hecker M, Murphy CA, Roesijadi G, Spade DJ, Spromberg JA, Wang M, Ankley GT. 2011. Adverse outcome pathways and ecological risk assessment: Bridging to population level effects. Environ Toxicol Chem 30:64-76.

54. Ankley GT, Bennett RS, Erickson RJ, Hoff DJ, Hornung MW, Johnson RD, Mount DR, Nichols JW, Russom CL, Schmieder PK, Serrano JA, Tietge JE, Villeneuve DL. 2010. Adverse outcome pathways: A conceptual framework to support ecotoxicology research and risk assessment. Environ Toxicol Chem 29:730-741.

55. Trudel M, Rassmussen J. 2006. Bioenergetics and mercury dynamics in fish: A modelling perspective. Can J Fish Aquat Sci 63:1890-1902.

56. Pickhardt PC, Stepanova M, Fisher NS. 2006. Contrasting uptake routes and tissue distributions of inorganic and methylmercury in mosquitofish (Gambusia affinis) and redear sunfish (Lepomis microlophus). Environ Toxicol Chem 25:2132-2142.

57. Fänge R, Grove D. 1979. Digestion. In Hoar WS, Randall DJ, Brett JR, eds, Fish Physiology, Vol 8. Academic, New York, NY, USA, pp 161260

58. Leaner JJ, Mason RP. 2002. Factors controlling bioavailability of ingested methylmercury to channel catfish and Atlantic sturgeon. Environ Sci Technol 36:5124-5129.

59. Goto D, Wallace WG. 2009. Influences of prey and predator dependent processes on cadmium and methylmercury trophic transfer to mummichogs (Fundulus heteroclitus). Can J Fish Aquat Sci 66:836846.

60. Leaner JJ, Mason RP. 2002. Methylmercury accumulation and fluxes across the intestine of channel catfish, Ictalurus punctatus. Comp Biochem Physiol C Toxicol Pharmacol 132:247-259.

61. Clarkson TW. 1997. The toxicology of mercury. Crit Rev Clin Lab Sci 34:369-403

62. Harris HH, Pickering IJ, George GN. 2003. The chemical form of mercury in fish. Science 301:1203.

63. George GN, Singh SP, Prince RC, Pickering IJ. 2008. Chemical forms of mercury and selenium in fish following digestion with simulated gastric fluid. Chem Res Toxicol 21:2106-2110.

64. Rabenstien DL, Evans CA. 1978. The mobility of methylmercury in biological systems. Bioinorg Chem 8:107-114.

65. Berntssen MHG, Hylland K, Lundbye AK, Julshamn K. 2004. Higher faecal excretion and lower tissue accumulation of mercury in Wistar rats from contaminated fish than from methylmercury chloride added to fish. Food Chem Toxicol 42:1359-1366. 
66. Bowling AM, Hammerschmidt CR, Oris JT. 2011. Necrophagy by a benthic omnivore influences biomagnification of methylmercury in fish. Aquat Toxicol 102:134-141.

67. Ralston NVC, Raymond LJ. 2010. Dietary selenium's protective effects against methylmercury toxicity. Toxicology 278:112-123.

68. Seppanen K, Soininen P, Salonen JT, Lotjonen S, Laatikainen R. 2004. Does mercury promote lipid peroxidation? An in vitro study concerning mercury, copper, and iron in peroxidation of low-density lipoprotein. Biol Trace Elem Res 101:117-132.

69. Turner MA, Swick AL. 1983. The Wabigoon River system: IV. Interaction between mercury and selenium accumulated from waterborne and dietary sources by northern pike (Esox lucius). Can J Fish Aquat Sci 40:2241-2250.

70. Chen Y-W, Belzile N, Gunn JM. 2001. Antagonistic effect of selenium on mercury assimilation by fish populations near Sudbury smelters? Limnol Oceanogr 46:1814-1818.

71. Dang F, Wang W-X. 2011. Antagonistic interaction of mercury and selenium in a marine fish is dependent on their chemical species. Environ Sci Technol 45:3116-3122.
72. Deng D-F, Teh F-C, The SJ. 2008. Effect of dietary methylmercury and seleno-methionine on Sacramento splittail larvae. Sci Total Environ 407:197-203

73. Wiener JG, Spry DJ. 1996. Toxicological significance of mercury in freshwater fish. In Beyer W, Hienz G, Redmon-Norton A, eds, Environmental Contaminants in Wildlife: Interpreting Tissue Concentrations. CRC, Boca Raton, FL, USA, pp 297-339.

74. Shore RF, Pereira G, Walker LA, Thompson DR. 2011. Mercury in nonmarine birds and mammals. In Beyer WN, Meador JP, eds, Environmental Contaminants in Biota: Interpreting Tissue Concentrations, 2nd ed. CRC, Boca Raton, FL, USA, pp 609-624.

75. Thompson DR. 1996. Mercury in birds and terrestrial mammals. In Beyer WN, Heinz GH, Redmond-Norwood AW, eds, Environmental Contaminants in Wildlife: Interpreting Tissue Concentrations. CRC, Boca Raton, FL, USA, pp 341-356.

76. Viera LR, Gravato C, Soares AMVM, Morgado F, Guilhermino L. 2009. Acute effects of copper and mercury on the estuarine fish Pomatoschistus microps: Linking biomarkers to behaviour. Chemosphere 76:14161427. 